\title{
XLIX. On a diagram of freezing-point depressions for electrolytes
}

\section{Prof. J.G. MacGregor F.R.S.}

To cite this article: Prof. J.G. MacGregor F.R.S. (1900) XLIX. On a diagram of freezing-point depressions for electrolytes, Philosophical Magazine Series 5, 50:306, 505-519, DOI: $10.1080 / 14786440009463940$

To link to this article: http://dx.doi.org/10.1080/14786440009463940

册 Published online: 21 Apr 2009.

Submit your article to this journal

Џ Article views: 7

Q View related articles $\sqsubset$ 
prevents the formation of oxygen and nitrogen compounds, thus blotting out some lines: and that it permits the formation of hydrogen compounds, thus accounting for the appearance of new lines.

Whatever the explanation may be, the following curious relation was found to exist between the arc and spark spectra, namely, all lines in the arre spectra which are affected by hydrogen, whether entanced or diminished, helong to the spark spectrum also.

To illustrate : the arc spectrum of tin in hydrogen shows two strong lines at $\lambda 3352 \cdot 15$ and $\lambda .3283 \% 31$, of which there is not the faintest trace in the ordinary tin arc. But these two are among the strong lines of the tin spark. See Hartley and Adeney's list. In like manner, I have photographed, on the same plate, the iron spark in air, the iron are in air, and the iron arc in hydrogen. In every case examined, the lines affected by hydrogen are spark-lines.

On the contrary, the lines which belong to Kayser and Runge's series are unaffected by the change from air to hydrogen.

If these series prove e jually stable in otber gases, this stability may form a criterion for dividing a spectrum into two groups-one of which will contain all the series lines, the other of which will contain none of them.

North-Western University,

Eranston, Illinois. July 1900 .

XLIX. On a Diayram of Freezing-point Inepressions for Electrolytes. By Prof. J. G. MacGregor, F.R.S., Dalhonsie College, IIalifax, N.S.*

\section{[Plate IV.]}

TWHE object of this paper is to describe a diagrammatic method of taking a bird's-eye view of such knowledge as we possess of the relation of the depression of the freezingpoint to the state of ionization in aqueons solutions of electrolytes, and to show that such diagrammatic study gives promise of throwing much light npon the following questions :-(1) $\dagger$ Has the depression-constant a common value for all electrolytes, and if so what is it? And (2) what is the state of association, and what the mode of ionization, of electrolytes in solution?

- Communicated by the Author. An abetract of a paper read before the Nova Scotirn Institute of Science.

+ On this question see also a paper recently communicated to the Royal Society of Caneda, and io be published in its Transactions for 1900. 
If an extremely dilute solution contain an electrolyte whose molecule as it exists in solution contains $p$ equivalents and dissociates into $q$ free ions, and if $a$ is its ionization-coefficient, and $k$ its depression-constant, the equivalent depression will be :

$$
\delta=\frac{k}{p}(1+\alpha(q-1)) .
$$

If, therefore, we plot a diagram of curves with ionizationcoefficients as ordinates, say, and equivalent depressions as abscissa, the resulting curves must at extreme dilution $(\alpha=1)$ be tangential to the straight lines represented by the above equation, provided the proper values of $k, p$, and $q$ be employed. These straight lines, which for shortness we may call the tangent lines of the curves, can readily be drawn in the diagram with any assumed value of $k$, and on any admissible assumptions as to the values of $p$ and $q$. In the diatgram (Pl. IV.) the broken lines are the tangent lines for the electrolytes examined, on various assumptions as to constitution in solution and mode of ionization, and for $k=1 \cdot 85$. They are indicated by the inscriptions $1-2,2-3, \& c$. , the first figure in each giving the number of equivalents in the molecule as it is assumed to exist in solution, and the second the number of free ions into which the molecule is assumed to dissociate. Thus 1-2 is the tangent line for an electrolyte such as $\mathrm{NaCl}$, on the assumption that it exists in solution in single molecules, each of which has therefore 1 equivalent and dissociates into 2 ions. If assumed to associate in double molecules with unchanged mode of ionization, its tangent line would be indicated by 2-4; and if the double molecules were assumed to dissociate into $\mathrm{Na}$ and $\mathrm{NaCl}_{2}$, by 2-2. The line for $\mathrm{H}_{2} \mathrm{NO}_{4}$ on the assumption that its molecules undergo no association and have thas 2 equivalents, and that they dissociate each into 3 ions, would be indicated by $2-3$.

In a few cases dotted lines have been introduced to show what the tangent lines would be with other values of $k, 1 \cdot 83$, $\& c .$, the constant used in such cases being indicated.

The curve for any given electrolyte must start at the intersection of its tangent line with the line $\alpha=1$, to which point we may refer for shortness as the intersection of its tangent line. What its form will be may be anticipated from the following theoretical considerations :-The equivalent depression in dilute solutions of non-electrolytes is proportional to

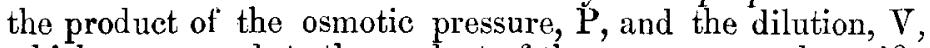
which corresponds to the product of the pressure $p$ and specific 
volume, $v$, in the case of a gas. If $p v$ is plotted against $v$, the resulting curve is convex towards the axis of $v$, and passes in general through a point of minimum value of $p v$. Hence if PV, and therefore equivalent depression, be plotted against $V$, we may expect to get curves of the same general form. And experiment shows that in some cases at least we do. As in the case of gases the variation of $p v$ is ascribed to the mutual action of the molecules and their finite volume, so in the case of solutions the variation of $\mathrm{PV}$ is attributed to similar disturbing influences.

Owing to ionization the curve of an electrolyte will differ from that of a non-electrolyte (1) because of the change thereby produced in the number of the molecules (including free ions) in unit of volume, and (2) because of the change produced in the disturbing influences referred to. The former change is doubtless the more important, and I shall assume the latter to be negligible, for the present purpose. Now dissociation increases continuously with dilntion. If therefore association of molecules does not occur, and if the mode of ionization does not change, the equivalent depression must be increased by the dissociation in a ratio which increases continuously with dilution. The change produced in the curve by dissociation, therefore, will be a shear parallel to the equivalent depressionaxis and increasing with dilution. The resulting curve will consequently remain convex towards the axis of dilution, but it will be less likely than the curve of a non-electrolyte to exhibit the minimum point.

If now we plot equivalent depression against ionizationcoefficient, instead of dilution, the result will be the same as if we shortened the dilution ordinates of the various points of the curve just mentioned, in ratios increasing with the dilution; which process must leave the curve convex towards what was the dilution-axis, but is now the ionization-coefficient axis.

If therefore no change occur in the association of molecules or in the mode of ionization, the curve of an electrolyte on the diagram must start at the intersection of its tangent line, tangentially to that line, and bend away from it, as dilution diminishes, to the right, possibly passing through a point of minimum equivalent depression. We may speak of such a curve as the normal curve for the tangent line corresponding to the given conditions as to constitution in solution and mode of ionization.

If, the constitution of the electrolyte in the solution remaining constant, the mode of ionization changes as dilution diminishes, say in such a way that the molecules dissociate 
on the average into a smaller number of ions, the equivalent depression will diminish more rapidly than it otherwise would. The curvature of the curve will therefore diminish and may possibly become zero and change sign, the curve thus becoming concave towards the ionization-coefficient axis and possibly crossing the tangent line. In such a case the curve will at the start coincide with the nomal curve of the tangent line determined by the initial conditions as to association and mode of ionization, and at the finish with the normal curve of the tangent line determined by the final conditions; and between the start and the finish it will gradually change from the one to the other.

If, as dilution diminishes, association of molecules into double or other multiple molecules occurs, the mode of ionization remaining the same, the equivalent depression will be thereby made to diminish more rapidly than it otherwise would, and the general effect on the form of the curve will be of the same kind as under the conditions just considered. But the normal curves of the tangent lines determined by the final conditions will be quite different in the two cases.

It follows that by plotting, so far as experiment allows, the curves of observed equivalent depression against ionizationcoefficient, and drawing in the tangent lines for different values of the depression-constant and on different assumptions as to association and mode of ionization, we may be able to determine, with a smaller or greater probability, what the state of association and the mode of ionization are, what are the tangent lines to whose intersections the curves would run out if observations at extreme dilution could be made, and what the values of the depression-constant are to which these lines correspond.

\section{Data for the Diagram.}

To draw the experimental curves, we must have corresponding values of the depression and of the ionizationcoefficient at the freezing-point, or what in most cases will be sufficiently near, at $\delta^{\circ} \mathrm{C}$. The former are obtained by direct measurement; but the latter only indirectly from conductivity observations. It is not of course known how closely the ionization-coefficients, even during the passage of the current, can thus be determined, or if the state of ionization during the passage of the current is to be regarded as being the same as when the current is not flowing. But as it has been shown that electrically determined coefficients enable us to predict, within the limit of error of observation, not only 
the conductivity and results of electrolysis* of moderately dilute complex solutions, but also their density, viscosity, and other non-electrical properties $t$, it would appear to be probable that for moderately dilute and very dilute solutions electrically determined coefficients are approximately exact, not only for a solution through which a current is passing, but generally.

The available data as to ionization-coefficients at $0^{\circ}$ are unfortunately few. Whetham $\ddagger$ has recently published some most valuable determinations, having measured the conductivity at $0^{\circ}$ of series of solutions down to extreme dilution, with what one may call appareil de luxe, and found the ratio of the equivalent conductivity to the maximum equivalent conductivity. For neutral salts his coefficients must inspire great confidence. But in the case of the acids they seem to me to be probably too bigh. For the maximum equivalent conductivity of an acid is probably lower than it would be, were it not for the disturbing influence, whatever it is, which makes the equivalent-conductivity-concentration curve not only reach but pass through a maximum point, as concentration diminishes.

Archibald and Barnes $\S$, working in my laboratory, measured the conductivity both at $0^{\circ}$ and $18^{\circ}$ for series of solutions down to dilutions at which the ratio of the two conductivities became constant; and assuming that the same ratio would hold at extreme dilution, they calculated the eqnivalent conductivity at extreme dilution for $0^{\circ}$ from Kohlrausch's values for $18^{\circ}$. They used this method only because appliances were not available with which observations at extreme dilution could be made. If the ratio mentioned really does become constant as dilution increases, the method is likely to give coefficients with too low or too high values according as the ratio at moderate dilutions diminishes or increases with dilution (it was found to increase with $\mathrm{KCl}$ and $\mathrm{K}_{2} \mathrm{SO}_{4}$ ). For it will probably become constant within the limit of error of observation before it has really reached constancy. And if it changes with dilution in a slightly wavy manner, even though on the whole tending to constancy, it may be regarded as having become constant when really passing through a. maximum or minimum point.

* Trans. Roy. Soc. Can. (2) iv. sec. 3, p. 117 (1898).

$\dagger$ See Phil. Mag. [5] xliii. pp. 40 \& 99 (1897); also Archibald, Trans. Nova Scot. Inst. Sci. ix. p. 335 (1897-98); and Barnes, ibid. x. pp. $49 \&$ $113(1899-1900)$.

† Zschr.f. phys. Chem. xxxiii. p. 344 (1900).

\$ Archibald, Trans. N. S. Inst. Sci. x. p. 33 (1898-9); Barnes, ibid. x. p. 189 (1899-1900); and Trans. Roy. Soc. Can. [2] vi. (1900).

Phil. Mag. S. 5. Vol. 50. No. 306. Nov. 1900. $2 \mathrm{~N}$ 
Déguisne's* observations on the variation of conductivity with temperature between $2^{\circ} \mathrm{C}$. and $34^{\circ}$ have enabled me, by the method just mentioned, to make rough determinations of the ionization-coefficients at $0^{\circ}$ in some cases, on the assumption that his empirical constants might be used down to $0^{\circ}$. According to Déguisne's observations the ratio of the conductivities at $0^{\circ}$ and $18^{\circ}$ usually changes gradually down to dilutions of 1000 litres per gramme-equivalent, and between that and 2000 undergoes rapid change. As observations at great dilution are attended by considerable difficulty, I have assumed that these sudden changes were probably due to errors of observation. If they were not, my Déguisne coefficients (for which Déguisne himself is of course not to be held responsible) may be considerably out.

In some cases I have obtained coefficients from the ahove data by extrapolation, in order to make use of available depression data. In such cases I have plotted side by side ionization-coefficient-concentration curves for both $0^{\circ}$ and $18^{\circ}$, using values for $18^{\circ}$ based on Kohlrausch's conductivities, and I have then produced the $0^{\circ}$ curve beyond the limit of observation under the guidance of the $18^{\circ}$ curve.

I need not give here the values of the coefficients used in the diagram. They will be found in the papert of which this is an abstract, and have been fairly accurately embodied in the accompanying diagram (Plate IV.).

I have used all the accessible observations of depression for the electrolytes for which data were available as to ionization at $0^{\circ}$, including observations by Arrhenius $\ddagger$, Raoult $\$$, Loomis\|, Jones $\|$, Abegg**, Wildermann $\dagger \dagger$, Ponsot $\ddagger$, Archibald $\S$, and Barnes $\S$. The methods used by these observers are for the most part well known. Archibald and Barnes used modified forms of Loomis's method. Arrhenius's obsel vations and some of Raoult's were made before important

* Temperatur-Coëfficienten des Leitvermögens sehr verdü̈nnter Lösungen: Dissertation, Strassburg; 1895 ; see also Kohlrausch u. Holborn, Leitvermögen d. Elektrolyte, Leipzig, 1898.

+ Trans. N. S. Inst. Sci. vol. x. Part 2 (not yet published).

Zschr.f. phys. Chem. ii. 491 (1888).

Ibid. ii. p. 501 (1888); and xxvii. p. 617 (1898).

II Phys. Review, i. pp. $199 \& 274$ (1893-4); iii. p. 270 (1896); and iv. p. 273 (1897).

q Zschr.f phys. Chem. xi. pp. 110 \& 529 (1893); and xii. p. 623 (1893).

** Tbid. xx. p. 207 (1896).

t† Ibid. xix. p. 233 (1896).

I Recherches sur la Congelation des Solutions Aqueuses: Paris, Gauthier-Villars (1896).

$\$ \S$ Loc. cit. 
improvements in freezing-point determinations had been recognized as necessary.

In cases in which there was but one series of observations available, I have plotted the actual observations in the diagram, though sometimes smoothing the curves a little. In cases in which two or more series were available, I first plotted the various observations and then drew mean curves, making them represent all the observations as well as I could, but giving greater weight to recent observations than to those of earlier date, and to long series of consistent observations than to short series or to series which were more erratic. These mean curves were then entered in the diagram.

The curves are labelled with the initial letters of observers' names ( $R$ for Raoult, \&c., A for Archibald, $A b$ for $A b e g g$ ), so as to show both the depression-observations on which they are based, and the ionization-coefficients used in plotting them. Thus the inscription $\mathrm{KCl}(\mathrm{J}-\mathrm{W})$ means that Jones's depressions and Whetham's coefficients were used; $\mathrm{H}_{2} \mathrm{SO}_{4}$ (JLB-B), that the curve is a mean curve based mainly at least on depression-observations by Jones, Loomis, and Barnes, and plotted with Barnes's coefficients. The limits of concentration for the curves are indicated also in gramme-equivalents per litre. The equivalent depressions plotted are in all cases depressions per gramme-equivalent in one litre of solution.

Some of the curves are entered on an inset drawn on four times the scale of the main diagram.

In interpreting the curves we must not only bear in mind what has been said above about the probable accuracy of the ionization-coefficients, but must in addition note the tendeney exhibited by the curves of the various observers, as dilation increases, to run off at great dilution in directions, characteristic of the observers, to the left or right relatively to the course pursued by them at moderate dilution. Thus Abegg's curves (see $\mathrm{NaCl}, \mathrm{KCl}, \mathrm{K}_{2} \mathrm{SO}_{4}$ ) and Jones's (see NaCl, KCl, $\mathrm{NH}_{4} \mathrm{Cl}$, and $\mathrm{HCl}$ ) run off to the right. So do Arrhenius's (not plotted), in a marked manner. Raoult's tendency is also to the right (see $\mathrm{NaCl}$; his $\mathrm{K}_{2} \mathrm{SO}_{4}$, not plotted, shows it also; his most dilute KCl observation he himself clearly regards as accidentally out). On the other hand, Loomis's curves (see $\mathrm{HCl}, \mathrm{KNO}_{3}, \mathrm{NH}_{4} \mathrm{Cl}, \mathrm{BaCl}_{2}$ ) go to the left. So do Ponsot's and probably Wildermann's (not plotted). And I gather from Ponsot's diagrams of Pickering's observations, to which I have not access, that Pickering's also have the leftward tendeney. Archibald's and Barnes's curves show $2 \mathrm{~N} 2$ 
less tendency to diverge than those of any other observers. And although this may be partially, it is not wholly, due to their having worked at moderate dilution only ; for in several cases, pointed out below, the curves of other observers start on a divergent course within their limit of dilution. But the fact that their curves usually agree with Loomis's would lead one to suspect them of a leftward tendency also.

The divergence, as shown on the diagram, is most marked in the case of highly dissociated electrolytes ( $\mathrm{NaCl}, \mathrm{HCl}, \& \mathrm{c}$.$) ,$ in which at great dilution the rate of increase of ionization with dilution is small, the curves being crushed up, therefore, into a small space. But it is obvious also in the $\mathrm{K}_{2} \mathrm{SO}_{\text {i }}$ curves (especially Abegg's) and the $\mathrm{BaCl}_{2}$ curves (including Ponsot's, not shown). And although for $\mathrm{MgSO}_{4}$ and $\mathrm{H}_{3} \mathrm{PO}_{4}$ whose ionization increases rapidly with dilution, the single curves do not reveal it, the relative positions of the two curves in each case are what they might be expected to be, if they were tending unduly, Jones's to the right and Loomis's to the left.

This tendency is explicable at once when we reflect that as it is equivalent depression that is plotted, the errors of the observations are brought into greater and greater prominence as dilution increases. According, therefore, as the characteristic error of an observer's method of measuring total depression is positive or negative, will his curves of equivalent depression diverge at great dilution to the right or left of their true course ; and they must diverge even if the error is very small.

The equivalent-depression curves of individual observers are therefore open to grave suspicion at high dilutions; and since one can never be sure that the errors of different methods will even approximately neutralise one another, mean high-dilution curves are not much more trustworthy than their components*. It is much safer, therefore, to base

* It is consequently inadmissible to proceed as Raonlt has done, in determining depression-constants (loc. cit. p. 658), viz., by selecting highdilution curves which are in agreement and applying extrapolation to a mean curve derived from them; for such procedure may involve the selection of observations made by methods which have characteristic errors of the same sign. In fact a mean curve based on observations which agree well at low dilution, but disagree markedly at high dilution, would be likely to give a better result, as nore probably combining observations with small characteristic errors of opposite sign. Raoult's procedure is open to other objections. For (1) his curves of equiralent depression against total depression make series of obselvations appear to be in greater disagreement than they really are, and are thus not belpful in making a judicious selection of observations to be used; and (2) extrapolation of such curves not only gives a result affected by the 
conclusions as to depression-constants on moderate-dilution curves, although the conclusions they admit of may not be so exact as we might wish.

\section{Discussion of the Curves.}

Electrolytes such as $\mathrm{KCl}, \mathrm{HNO}_{3}$, $\mathrm{KOH}$, have 1 equivalent in the single molecule and 2 ions. If, therefore, they exist in solution in single molecules, their curves will be normal 1-2 curves. If their molecules are all double or triple, the curves shonld be $2-4$ or 3-6 curves, provided association does not involve change in the mode of ionization. If it does, they may be $2-3$ or $3-4,3-3$ or $3-2$ curves respectively, according to the change that may occur. If the molecules are single at extreme dilution, but become double or triple as dilution diminishes, the curves should start as 1-2 curves and undergo the appropriate transformation.

The electrolyte for which we have the most complete and trustworthy data is $\mathrm{KCl}$. The LB-B curve (see main diagram) is based for the most part on two series of observations in close agreement and by methods exhibiting less divergence than the others. Jones's curve runs a little to the right of it; Abegg's a little to the left. Both Raoult's and Wildermann's cross it, the latter being somewhat steeper, the former less steep. Ponsot's coincides with the lower part of it, but in the upper part diverges to the left. In form the curve is thus probably trustworthy ; but, being plotted with Barnes's coefficients, it may be too high or too low. The R-W and $J-W$ curves (see inset), are not open to this snspicion, but at the dilutions to which even their lower and more trustworthy parts apply they may have begun to diverge unduly rightwards. If the LB curve is plotted with Whetham's coefficients (extrapolated) it comes into a position (see LB-W curve on inset) to the left of the $R-W$ and $J-W$ curves, the usual relative position of the curves of these observers. Loomis's own curve for somewhat greater dilutions than those of the LB-W curve, when plotted with Whethan's coefficients, coincides very nearly with the boundary-line of the inset. Wildermann's is a little to the left of Loomis's, and somewhat steeper. Both exhibit a slight rightward bending, as do all the others.

average of the characteristic errors of the observations used, but also neglects the possibility, in some cases the probability, that owing to change in association and mode of ionization the law of the change of curvature may be very different beyond the limits of observation from what it is within these limits. 
It would be difficult to draw a mean curve with confilence; but any such curve would run about midway between the 1-2 and 2-4 (1-85) lines, would have a slight rightward bending at its upper end, and if produced with diminisling curvature would run out to a point a little to the right of the $1-2(1.85)$ intersection.

If this intersection were the starting-point of the curve and if there were no association, the curve should lie wholly to the right of the 1-2 (1.85) line. If, as dilution diminished, sufficient doubling of molecules with unchanged mole of ionization should occur, the curve, after first bending away from that line to the right, would change its curvature, bend towards the line and cross it, and then run toward: and finally away from the 2-4 line, as the mean curve appears to do.

As the 2-2 line is far to the left, the mean curve might be accounted for also on the assumption of a very slight formation of double molecules dissociating into two ion:-a formation of such molecules so slight as probably to involve no greater variation of the migration numbers with concentration than has been observed.

If the 1-2 (1.86) intersection be assumed as the startingpoint of the curve, the mean curve would cut the $2-4(1.86)$ line. If, therefore, association in molecules with unchringed mode of ionization were assumed, some formation of triple molecules would be indicated, and if the a-sociated molecules were assumed to dissociate into two free ions, a greater extent of such association would be indicated. Thus with this starting-point less probable assumptions as to association must be made to account for the observations.

If the 1-2 (1.84), or even the 1-2 (1.845) intersection were taken as the starting-point, the curve must bend considerably to the left before running out, of which bending none of the experimental curves give any indication whatever.

The most probable conclusion then that we can draw from the observations is that the depression-constant is $1 \cdot 85$, with a limit of error of $\cdot 01$ or perhaps $\cdot 005$, that the clectrolyte has single molecules at great dilution, and that as dilurion diminishes either double molecules with unchanged mode of ionization form to a considerable extent, or double molecules dissociating into two ions, to a small extent.

Loomis's and Barnes's observations on which the $\mathrm{NaCl}$ (LB-B) curve is mainly based are also in close agreement; but as Loomis's curve for slighily greater dilution bends slightly to the right, the upper part of the LB-I3 curve 
should probably have greater curvature. Jones's curve for moderately dilute solutions runs a little to the left of it, and at higher dilutions diverges markedly to the right (as separately shown). Abegg's observations are on both sides of it, but at higher dilutions his curve also goes to the right, as shown. Raoult's tonches it, but goes off to the right. Arrhenius's (not shown) is considerably to the right throughout, and goes widely rightward at greater dilutions. Ponsot's is a little to the left. As the LB-B curve is plotted with Barnes's coefficients, it is probably too low. If it be raised about $2 \cdot 5$ per cent., the amount by which Whethan's KCl coefficients are higher than Barnes's, it will lie along the 1-2 (1.85) line, or a little above or below that line, with its upper end so directed as to run out probably at a point nearer the 1-2 (1.85) intersection than either the 1-2 (1.83) or the 1-2 (1.87) intersection. The defective data as to ionization prevent our drawing a more definite conclusion than that the association indicated, if any, is less than in the case of $\mathrm{KCl}$, and that the depression-constant is 1.85 with a limit of error of perhaps 0.02 .

The $\mathrm{HCl}$ curve is interesting as exhibiting a point of minimum equivalent depression. The observations on which the LB-B curve is based are in good agreement. Jones's curve almost coincides with it in the lower part, but goes off to the right in the upper part and at higher dilutions, as shown separately. Loomis's curve at higher dilutions, also separately shown, goes off to the left, but in a less marked manner. As drawn, the upper part of the mean curve lies between the 1-2 and 2-4 (1.85) lines, and it is running out to a point a little beyond the 1-2 (1.86) intersection (see inser). But as it is plotted with Barnes's coefficients it is perbaps too low. If raised from 0 to 2 per cent. it might run out at any point between the 1-2 (1.81) and 1-2 (1.86) intersections. The data are of course very defective, but they are consistent with a depression-constant of about 1.85 , and they seem to indicate a greater extent of association than in the case of $\mathrm{KCl}$.

The $\mathrm{L}-\mathrm{D}$ and $\mathrm{J}-\mathrm{D}$ curves for $\mathrm{NH}_{4} \mathrm{Cl}$ (see inset) are not in agreement, having the usual relative position of $\mathrm{L}$ and $J$ curves. A mean curve based on their lower parts would be slightly to the left of the 1-2 (1.85) line, and directed to a point considerably to the right of the 1-2 (1.86) intersection. It might thus indicate anything between a high value of the depression-constant, accompanied by very considerable association of molecules, and a constant of about 1.85 without association in dilute solutions, and with only a slowly increasing association in stronger solutions. 
The $\mathrm{HNO}_{3}$ curve (see inset) is a mean curve based on Loomis's and Jones's. Both are beyond the bounds of the inset, the former to the left and the latter to the right. Neither this curve nor that of $\mathrm{KNO}_{3}$ is sufficiently trustworthy to warrant any close inspection ; but both are clearly consistent with the 1.85 value of the depression-constant. If the leftward bending of the $\mathrm{KNO}_{3}$ curve in its lower part were actual, as well as the position of the curve, the formation of triple molecules might be indicated. But being a Loomis curve, it is open to the suspicion of being as a whole too far to the left, and it is plotted with doubtful coefficients.

The KOH curves, Loomis's on the main diagram and Jones's on the inset, are useful only to illustrate the difficulty of making concordant observations by different methods. As usual, Loomis's is to the left and Jones's to the right.

Electrolytes such as $\mathrm{BaCl}_{2}, \mathrm{H}_{2} \mathrm{SO}_{4}, \mathrm{Na}_{2} \mathrm{CO}_{3}$ have 2 equivalents in the single molecule which may dissociate into 3 or into 2 ions. If there is no association, they will therefore have 2-3 or 2-2 curves according to the mode of ionization. If there is complete donbling of molecules, the curves will be 4-6 or 4-4 curves, provided the doubling does not involve change in mode of ionization. Otherwise they might be $4-5,4-3$, or 4-2 curves. If the molecules are associated in threes, the curves will be 6-9 or 6-6 curves, with the above proviso.

Both Loomis's and Jones's curves for $\mathrm{BaCl}_{2}$ are shown on the diagram, plotted with Whetham's coefficients (rough extrapolated values, however, in the case of the former). Ponsot's curve agrees very closely with Loomis's. Bearing in mind the rightward and leftward tendencies of Jones's and Loomis's curves respectively, we may conclude from the curves of the diagram that the actual curve runs down to the right of the 2-2 line, bending away from it to the right, and that it would intersect the $\alpha=1$ line at a point between the $2-3(1.85)$ and the 2-3 (1.87) intersections, probably nearer the former than the latter. The curve is thus, so far as we can judge, a normal $2-3(1 \cdot 85 \pm \cdot 01)$ curve, running however very close to the 2-3 line. The diagram therefore indicates that $\mathrm{BaCl}_{2}$ exists in solution in single molecules, dissociating into three ions, at least for the most part, and that it has a depression-constant nearer 1.85 than 1.87 .

The $\mathrm{H}_{2} \mathrm{SO}_{4}(\mathrm{~J}-\mathrm{W})$ curve for high dilutions being a Jones curve, is probably too far to the right, and, being plotted with Whetham's coefficients, is probably too high. Wildermann's curve for high dilutions runs parallel to it, considerably to the left. The JLB-B curve for lower dilutions is very nearly coincident with Barnes's curve, and in its lower 
part with Jones's and Loomis's as well. But in the upper part Jones's curve goes off markedly to the right, and Loomis's markedly to the left. Wildermann's is slightly to the left at the lower end, and diverges somewhat leftwards in the upper part. Ponsot's runs nearly parallel to it somewhat to the left, and diverges to the left at higher dilutions. The JIA-B curve is thus trustivorthy in form, but being plotted with Barnes's coefficients it is possibly too low. The actual rurve would thus appear to cross the 2-3 (1.85) line not far from its starting-point, bend towards the 4-6 (1.85) line, and run down below that line, finally hending slightly towards it again. Its course is therefore what it would be if it started as a $2-3$ curve for $k=1.85$ or thereahout, changed its curvature at a somewhat early stage, and tended to be transformed slowly into either a double-molecule curve or a $2-2$ curre, or perhaps hoth at once. The dia gram would therefore indicate that at extreme dilution $\mathrm{H}_{2} \mathrm{SO}_{4}$ exists in solution in single mulecules dissociating into three ions; that at an early stage and in a somewhat marked manner, either doubling of molecules sets in, or partial dissociation into two ions or perhaps both ; that the change increases slowly and steadily as dilution diminishes, and that at a concentration of ahout $0 \cdot 6$, if the coefficients at this concentration are to be trusted, the change is increasing in rate; also that the depression-constant may quite readily be about 1.85 .

The $\mathrm{K}_{2} \mathrm{SO}_{4}$ (LJA- $\Lambda$ ) curve is based on series of observaltions which in the main are in good agreement. It very nearly coincides with the Loomis and Archibald curves, and Ponsot's (not shown) runs down slightity to the left. In its lower part it coincides with the Jones curve, but in its upper part Jones's curve (separately represented for great dilutions) runs off to the right. Abegg's curve for lighter dilutions runs much more markedly to the right, though it is farther to the left at its lower end. Arrhenius's (not shown) is considerably to the right and diverges widely rightwards. The $\mathrm{Na}_{2} \mathrm{SO}_{4}(\mathrm{LA}-\mathrm{A})$ curve is also almost coincident with both the Loomis and the Archibald curves. Both Raoult's and Arrhenius's (neither shown) are considerahly to the right and diverge slowly rightwards. Such of these curves as are entered on the diagram, being plotted with Archibald's coefficients, are probably too bigh or too low, as the cuse may be. Those for great dilutions are too discordant to admit of discussion. The mean curves for both salts have the same general form. They run down, as drawn, a little below the z-3 (1.85) line. Their upper ends are so directed as to suggest their running out at the $2-3(1 \cdot 85)$ intersection or 


\section{On Freezing-point Depressions for Electrolytes.}

thereabout. At their lower ends they turn sharply to the left and eross the $2-3$ line, going toward the region of the dounte. molecule curves or of the $2-2$ curve. The turns are too slarp and the 4-6 and 6-9 lines too near to make their transtormation probable into double or triple molecule curres with unchanged mode of ionization. The diagran suggests rather their transtomation into $4-5,4$, or 2 or $2-2$ curves. If this be accepted, it means that at extreme dilution these sulphates exist in solution in single molecules dissociating into three ions, that partial dissociation into two ions or doubling of molecules sets in apparently at an early stace, but increases more slowly than in the case of $\mathrm{H}_{2} \mathrm{SO}_{4}$ until the dilution has been considerably diminisbed, when it undergoes rapid increase. A close determination of the depresion-constant cannot be made; but even if the curves have to be either raised or lowered a little and if, Loomis's tendency being leftward, their upper ends have to be shifted someiwhat to the right, they will be consistent with its being about 1.85 .

The $\mathrm{Na}_{2} \mathrm{CO}_{3}$ curves are too discordant to form a basis for discussion. But either Loomis's curve or a mean curve, or even Jones's curve itself is quite consistent with a depression constant of about 1.85 ; and both curres indicate the occurrence of rapid association or of rapid change of mode of ionization after considerable diminution of dilution. The fact that Loomis's curves tend towards the left, suggests that the actual curve after starting at the 2-3 interrection may bend considerably to the right before association or change of mode of ionization has advanced sufficientiy to change the direction of its curvature.

An electrolyte such as $\mathrm{MgSO}_{4}$, according as it may exist in solution in single, double, or triple molecrles, and according to their mode of ionization, may bave a $2-2,4-4$ or 2 , or i-6, 4,3 or 2 curve. Jones's curve lies to the right of the 2-2 $(1.85)$ line, bending towards it, and may quite readily be a $2-2(1.85)$ curve changing to a $4-4$ or $4-2$ curve. Loomis's lies berween the 2-2 and 4-4 lines. A mean curve would, at a concentration 02 , have already crossed the $2-2$ line. The data, such as they are, are consistent with the depression-constant having a value of about $1 \cdot 85$, and would indicate single molecules in dilute solutions, doubling of molecules at a very early stage, and a steady increuse in association throuvhont.

According as $\mathrm{H}_{3} \mathrm{PO}_{4}$, if it exist in solution in single molecules, may dissociate into 4,3 , or 2 ions, will it have al $3-4$, $3-3$, or 3-2 curve. If it have double molecules, its curve may be a $6-8,6-7$, \&c. to $6-2$ curve, according to the mode of ionization. Jones's curve, far to the left of the $3-4$ and $3-3$ 
lines, runs down to the right of the 3-2 (1.85) line, bending towards the line. IJoomis's lies between the 3-2 and 6-4 lines. A mean curve would be just to the right of the 3-2 line, and might readily run out at the $3-2(1 \cdot 85)$ intersection. This would indicate single molecules in dilute solutions dissociating into two ions, an early occurrence of doubling of molecules, and steady increase in the extent of association as dilution diminished, the double molecules formed dissociating into 4,3 , or 2 ions, but not into more. Although the coefficients with which the curves are plotted are doubtful, the curves are so nearly parallel to the axis of coefficients, that even a considerable error in their determination would not affect the above result.

\section{General Conclusions.}

Although the observations on which the above discussion is based are defective, and the particular conclusions drawn are consequently tentative, I think it may be held with some confidence: (1) that the curves of equivalent depression against ionization-coefficient have positions, forms, and slopes such as they might be expected to have on reasonable assumptions as to mode of ionization and constitution in solution, according to the Van 't Hoff-Arrhenius theory of the depression of the freezing-point in solutions of electrolytes; $(2)$ that they are consistent with the depression-constant having a common value of about $1.8 \tilde{5}$ for all the electrolytes examined, and that in the case of the electrolyte for which we have the best data, its curve is not consistent with a greater limit of error in this value than about 0.01, unless improbable assumptions are made with respect to the constitution of the electrolyte in solution; and (3) that the diagram enables us to reach in some cases conclusions of considerable probability with respect to the constitution of the electrolyte in solution and its mode of ionization.

L. The Motion of a Sphere in a Viscous Fluid. $B y$ H. S. AlLeN, M.A., B.Se.

[Continued from p. 338.]

[Plates I. \& II.]

III.

\section{Photographic Method of Determining Velocities.}

T $\mathrm{T}$ was only possible to apply the method already described 1 to cases in which the terminal velocity was small. In order to extend the range of observation it was decided to have recourse to a photographic method. Several ways of 


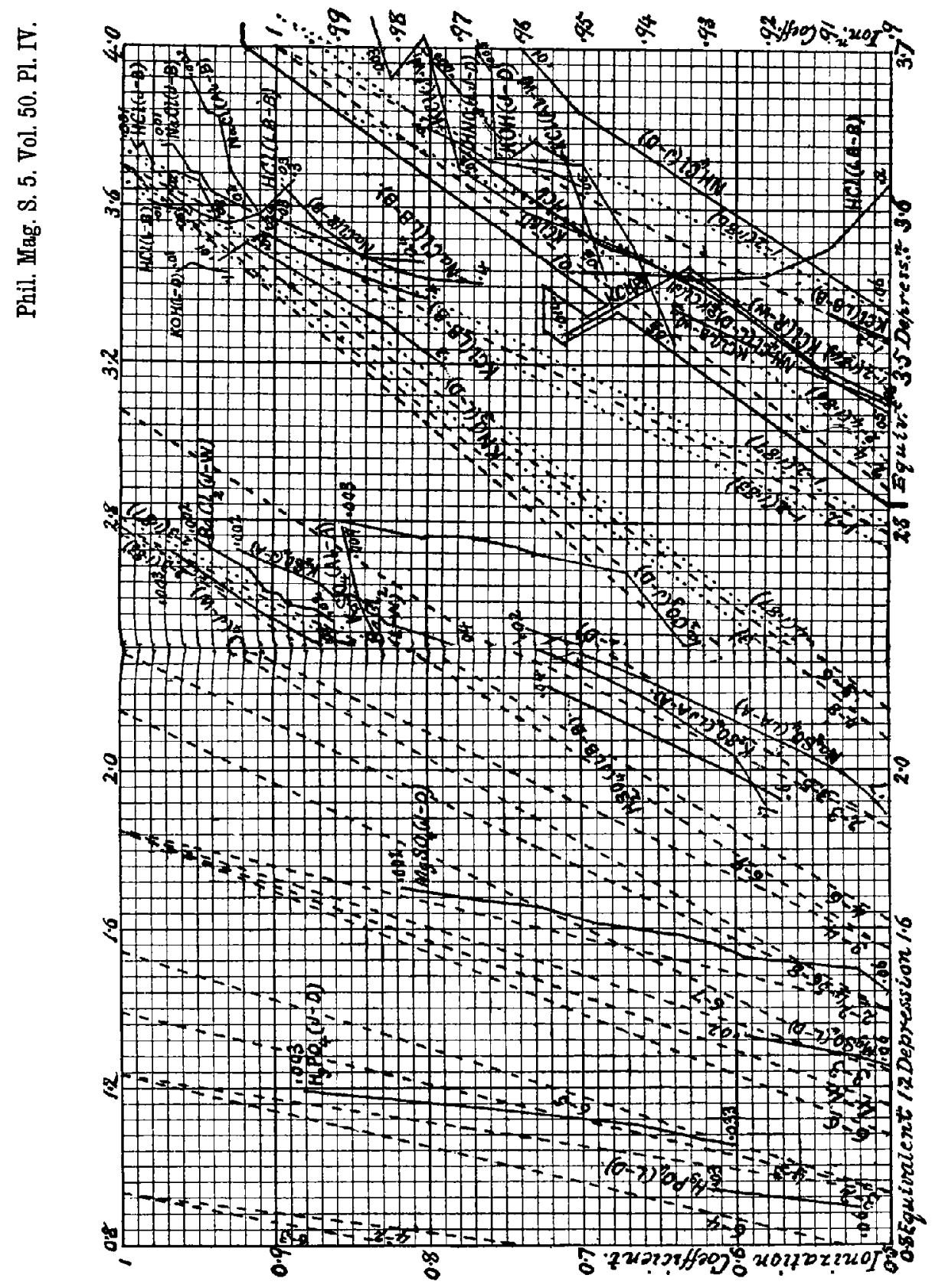

